\title{
Caracterização de profissionais de enfermagem que atendem pessoas com úlceras vasculares na rede ambulatorial
}

\author{
Characterization of nursing professionals who care for people with vascular ulcers in outpatient facilities \\ Caracterización de profesionales de enfermería que atienden a personas con úlceras vasculares \\ en la red ambulatoria
}

\section{Adriana Cristina de Santana', Maria Márcia Bachion', Suelen Gomes Malaquias", Flaviana Vieira', Diana Alves Carneiro', Jacqueline Rodrigues de Lima'}

\author{
' Universidade Federal de Goiás, Faculdade de Enfermagem e Nutrição, \\ Programa de Pós-Graduação em Enfermagem. Goiânia-GO, Brasil. \\ "Universidade Federal de Goiás, Faculdade de Medicina, \\ Programa de Pós-Graduação em Ciências da Saúde (Doutoranda). Goiânia-GO, Brasil.
}

Submissão: 02-04-2013 Aprovação: 14-10-2013

\section{RESUMO}

Objetivou-se descrever características demográficas e profissiográficas dos trabalhadores de enfermagem que atendem pessoas com úlceras vasculares, em salas de curativos da rede municipal de saúde. Trata-se de pesquisa transversal, descritiva, de abordagem quantitativa realizada numa grande cidade da região Centro-Oeste do Brasil, de setembro de 2010 a fevereiro de 2011 . Utilizou-se na coleta de dados um questionário para obtenção das características de interesse. Realizou-se análise descritiva e teste qui quadrado ( $p<0,05)$. Participaram 218 profissionais, dos quais 58,3\% nunca realizaram atividades de atualização em avaliação e tratamento de feridas, 40,7\% relataram pouca experiência na área, 61\% eram técnicos e auxiliares de enfermagem. Aproximadamente 10\% dos enfermeiros atuavam de modo fixo nas salas de curativos; os demais participavam do atendimento quando chamados. Os dados obtidos indicam fragilidades no processo de atendimento de enfermagem a pessoas com úlceras vasculares.

Descritores: Cuidados de Enfermagem; Úlcera da Perna; Úlcera Varicosa.

\section{ABSTRACT}

The purpose of the study was to describe the demographic and professional competence characteristics of the nursing workers who treat people with vascular ulcers in the dressing rooms of the municipal healthcare network. This cross-sectional, descriptive and quantitative study was performed in a large city in Midwestern Brazil, from September of 2010 to February of 2011. Data collection was performed using a questionnaire, and the data were subjected to descriptive analysis and qui square test $(p<0,05)$. The participants were 218 workers, $58.3 \%$ of which had never been involved in update activities related to wound evaluation and treatment, $40.7 \%$ reported having little experience in this field, $61 \%$ were nursing technicians and nurses' aides. Approximately $10 \%$ of the nurses worked exclusively in the dressing rooms, and the others helped in when called. The collected data indicate weaknesses in the nursing care delivered to people with vascular ulcers.

Key words: Nursing Care; Leg Ulcer; Varicose Ulcer

\section{RESUMEN}

Se objetivó describir características demográficas y profissiográficas de trabajadores de enfermería que atienden personas con úlceras vasculares, en salas de curaciones de la red municipal de salud. Esta investigación transversal, descriptiva, de abordaje cuantitativo, fue realizada en gran ciudad del Centro-Oeste de Brasil, de setiembre 2010 a febrero 2011. Datos recolectados mediante cuestionario enfocado a puntos de interés. Se efectuó análisis descriptivo y teste qui-quadrado $(p<0,005)$. Participaron 218 profesionales, de los que 58,3\% nunca había realizado actividades de atención en evaluación y tratamiento de heridas, $40,7 \%$ refieren poca experiencia en el área, $61 \%$ eran técnicos y auxiliares de enfermería. Aproximadamente $10 \%$ de enfermeros ostentaban cargo fijo en las salas de curaciones, el resto participaba de la atención por solicitud. Los datos indican fragilidades en el proceso de atención de enfermería a personas con úlceras vasculares.

Palabras clave: Atención de Enfermería; Úlcera de la Pierna; Úlcera Varicosa. 


\section{INTRODUÇÃO}

Estima-se que 1\% da população tenha úlceras vasculares. Entre estas lesões, as mais frequentes são as úlceras de origem venosa, que podem representar até $90 \%$ dos casos. É importante que os profissionais disponham de preparo adequado para o atendimento a essa população ${ }^{(1-3)}$.

A assistência às pessoas com lesões de pele exige abordagem holística, planejamento de estratégias e de intervenções que possibilitem alcançar os objetivos propostos ${ }^{(4)}$. O enfermeiro é responsável pelo tratamento e prevenção de feridas, devendo, no contexto de suas competências profissionais, avaliá-las, prescrever os cuidados e o tratamento mais adequado, orientar o paciente e/ou seus familiares, supervisionar a sua equipe na realização de curativos e possuir conhecimento científico e habilidades no manejo das lesões ${ }^{(5)}$.

Pesquisas de enfermagem sobre úlceras vasculares descrevem a ocorrência e as características das lesões ${ }^{(6)}$, as características da população que sofre esse tipo de agravo ${ }^{(7)}$, avaliam resultado da utilização de produtos na cicatrização das lesões ${ }^{(8)}$, mas, pouca atenção tem sido dada à investigação das características da força de trabalho que é responsável pelo atendimento às pessoas com esse tipo de feridas ${ }^{(9)}$.

Diante deste contexto, esta pesquisa teve como objetivos analisar as características demográficas e profissiográficas dos profissionais de enfermagem no atendimento as pessoas com úlceras vasculares, em salas de curativo da rede de atenção primária municipal, em uma grande cidade.

\section{MÉTODO}

Trata-se de um estudo transversal, descritivo e de abordagem quantitativa, realizado de setembro de 2010 a fevereiro de 2011, nas 40 unidades de saúde que possuem salas de curativo, onde ocorre atendimento de enfermagem às pessoas com úlceras vasculares, numa grande cidade da região Centro-Oeste.

A população desta pesquisa consistiu de 266 profissionais de enfermagem que, independentemente do sexo, idade e tempo de atuação na unidade, prestavam atendimentos às pessoas com úlcera vascular.

$\mathrm{Na}$ fase inicial de recrutamento, identificou-se que 15 $(5,63 \%)$ profissionais de enfermagem se encontravam de licença médica, duas $(0,74 \%)$ de licença por outros motivos e $11(4,13 \%)$ de férias. Foram então convidados para o estudo 238 profissionais, dentre os quais $20(8,4 \%)$ se recusaram a participar, sem motivos específicos. Assim, foram incluídos no estudo 218 profissionais de enfermagem que aceitaram participar e manifestaram sua anuência assinando o Termo de Consentimento Livre e Esclarecido.

O instrumento de coleta de dados, previamente refinado por um conjunto de cinco expertos, foi um questionário contendo itens relativos às características demográficas, profissiográficas e de experiência na área de tratamento de úlceras vasculares.

Os dados da pesquisa foram organizados em banco de dados em planilha Excel e posteriormente transferidos e analisados pelo software Statistical Package of Social Sciences for Windows ${ }^{\circledast}$, versão 17.0 , o qual permitiu a realização de estatística descritiva com distribuição de frequências simples e percentual e medidas de tendência central (mediana, média) e dispersão (desvio padrão) dos dados de caracterização demográfica e profissional. Utilizou-se o teste qui quadrado para analisar diferenças de proporções, mediante dicotomização das variáveis de interesse.

Este estudo foi aprovado pela Secretaria Municipal de Saúde do município estudado e pelo Comitê de Ética e Pesquisa da Universidade Federal de Goiás (Protocolo nº 101/2010).

\section{RESULTADOS}

Dos 218 profissionais de enfermagem, 85 (39,0\%) atuavam nas unidades como enfermeiros, 97 (44,5\%) como técnicos de enfermagem e $36(16,5 \%)$ como auxiliares de enfermagem.

O gênero feminino foi predominante, tanto entre técnicos e auxiliares como entre os enfermeiros, totalizando 201 profissionais (92,2\%). A idade variou entre 22 a 62 anos, com a mediana de 40 anos e média de 40,7 anos (DP $\pm 10,6$ ); a faixa etária mais frequente foi a de 49 anos ou mais para técnicos e auxiliares, enquanto que para a enfermeiros a faixa etária com maior número de participantes foi de 26 a 33 anos (Tabela 1). Considerando a faixa até 41 anos, o contingente de enfermeiros foi de $65,9 \%$, enquanto que os técnicos somaram $45,9 \%$. O grupo de enfermeiros era mais jovem, sendo a diferença significativa $(p=0,003)$.

Em relação ao tempo de atuação profissional, predominou entre os técnicos e auxiliares o período $\geq 20$ anos, enquanto para enfermeiros a frequência maior é de atuação profissional há um tempo menor que cinco anos (Tabela 1). Apesar desse longo tempo de formação, dos técnicos e auxiliares, 68,4\% atuavam na unidade, onde estavam lotados no momento, há um período menor que cinco anos. Considerando a proporção de enfermeiros e técnicos/auxiliares de enfermagem que apresentam o tempo de atuação profissional de até 5 anos, não houve diferença significativa $(p=0,052)$.

Aproximadamente $70 \%$, tanto de enfermeiros como técnicos e auxiliares atuavam nas salas de curativo, prestando atendimento às pessoas com úlceras vasculares há um período de tempo de até cinco anos. A escala de trabalho para atuação na sala de curativo variou de diária, semanal, mensal, trimestral e fixa. Assim, havia profissionais que prestavam atendimento no local desde diariamente até uma vez a cada três meses.

Oito $(9,4 \%)$ enfermeiros atuavam de modo fixo na sala de curativo, no cuidado direto às pessoas com úlceras vasculares e $77(90,6 \%)$ referiram comparecer à sala de curativos apenas para avaliar os casos, conforme solicitação dos demais profissionais e/ou para realizar orientações às pessoas com úlceras vasculares. Dessa maneira, toda a assistência de enfermagem era realizada predominantemente por auxiliares e/ou técnicos de enfermagem.

Como houve variação da escala de local de atendimento pelos profissionais, aproximadamente $20 \%$ de técnicos e auxiliares de enfermagem e $15 \%$ de enfermeiros estiveram prestando atendimento às pessoas na sala de curativo no dia da coleta de dados da pesquisa ou no dia anterior. A maioria participou dessa atividade havia mais de um mês (Tabela 2). 
Tabela 1- Distribuição dos profissionais de enfermagem $(n=218)$ de acordo o sexo, faixa etária, tempo de atuação profissional, tempo de atuação na unidade e na sala de curativos em um município de grande porte da região Centro-Oeste, set. 2010 a fev. 2011.

\begin{tabular}{|c|c|c|c|c|c|c|}
\hline \multirow[t]{2}{*}{$\begin{array}{l}\text { Características dos } \\
\text { profissionais de } \\
\text { enfermagem }\end{array}$} & \multicolumn{2}{|c|}{$\begin{array}{c}\text { Auxiliar ou } \\
\text { técnico de } \\
\text { enfermagem } \\
n=133 \\
\end{array}$} & \multicolumn{2}{|c|}{$\begin{array}{l}\text { Enfermeiro } \\
\quad n=85\end{array}$} & \multicolumn{2}{|c|}{$\begin{array}{c}\text { Total } \\
n=218\end{array}$} \\
\hline & $n$ & $\%$ & $n$ & $\%$ & $n$ & $\%$ \\
\hline \multicolumn{7}{|l|}{ Sexo } \\
\hline Feminino & 121 & 91,0 & 80 & 94,1 & 201 & 92,2 \\
\hline Masculino & 12 & 9,0 & 5 & 5,9 & 17 & 7,8 \\
\hline Total & 133 & 100,0 & 85 & 100,0 & 218 & 100,0 \\
\hline \multicolumn{7}{|l|}{ Faixa etária } \\
\hline $18-25$ anos & 7 & 5,3 & 9 & 10,6 & 16 & 7,3 \\
\hline $26-33$ anos & 21 & 15,8 & 27 & 31,8 & 48 & 22,0 \\
\hline $34-41$ anos & 33 & 24,8 & 20 & 23,5 & 53 & 24,3 \\
\hline $42-48$ anos & 24 & 18,0 & 11 & 12,9 & 35 & 16,1 \\
\hline 49 anos ou mais & 48 & 36,1 & 18 & 21,1 & 66 & 30,3 \\
\hline Total & 133 & 100,0 & 85 & 100,0 & 218 & 100,0 \\
\hline \multicolumn{7}{|l|}{$\begin{array}{l}\text { Tempo de atuação } \\
\text { profissional }\end{array}$} \\
\hline$<5$ anos & 26 & 19,5 & 30 & 29,4 & 56 & 25,7 \\
\hline $5 \vdash 10$ anos & 27 & 20,3 & 13 & 15,3 & 40 & 18,3 \\
\hline $10 \vdash 15$ anos & 22 & 16,5 & 16 & 18,8 & 38 & 17,4 \\
\hline $15 \vdash 20$ anos & 16 & 12,0 & 9 & 10,6 & 25 & 11,5 \\
\hline$\geq 20$ anos & 42 & 31,6 & 17 & 20,0 & 59 & 27,1 \\
\hline Total & 133 & 100,0 & 85 & 100,0 & 218 & 100,0 \\
\hline \multicolumn{7}{|l|}{$\begin{array}{l}\text { Tempo de atuação } \\
\text { na unidade de saúde }\end{array}$} \\
\hline$<5$ anos & 91 & 68,4 & 59 & 69,4 & 150 & 68,8 \\
\hline $5 \vdash 10$ anos & 17 & 12,8 & 20 & 23,5 & 37 & 17,0 \\
\hline $10 \vdash 15$ anos & 10 & 7,5 & 2 & 2,4 & 12 & 5,5 \\
\hline $15 \vdash 2$ anos & 9 & 6,8 & 3 & 3,5 & 12 & 5,5 \\
\hline$\geq 20$ anos & 1 & 1,2 & 6 & 4,5 & 7 & 3,2 \\
\hline Total & 133 & 100,0 & 85 & 100,0 & 218 & 100,0 \\
\hline \multicolumn{7}{|l|}{$\begin{array}{l}\text { Tempo de atuação } \\
\text { na sala de curativos }\end{array}$} \\
\hline$<5$ anos & 93 & 69,9 & 63 & 74,1 & 156 & 71,6 \\
\hline $5 \vdash 10$ anos & 16 & 12,0 & 16 & 18,8 & 32 & 14,7 \\
\hline $10 \vdash 15$ anos & 11 & 8,3 & 2 & 2,4 & 13 & 6,0 \\
\hline $15 \vdash 20$ anos & 10 & 7,5 & 3 & 3,5 & 13 & 6,0 \\
\hline$\geq 20$ anos & 3 & 2,3 & 1 & 1,2 & 4 & 1,8 \\
\hline Total & 85 & 100,0 & 133 & 100,0 & 218 & 100,0 \\
\hline
\end{tabular}

Tabela 2 - Data do último atendimento às pessoas com úlceras vasculares referida pelos enfermeiros, técnicos/auxiliares, $(n=218)$ que atuam nas salas de curativo da rede municipal em uma grande cidade da região Centro-Oeste, set. 2010 a fev. 2011.

\begin{tabular}{|c|c|c|c|c|c|c|c|}
\hline \multirow[b]{2}{*}{$\begin{array}{l}\text { Função na } \\
\text { unidade }\end{array}$} & \multicolumn{7}{|c|}{ Último atendimento à pessoa com úlcera vascular } \\
\hline & $\begin{array}{l}\text { Hoje } \\
n(\%)\end{array}$ & $\begin{array}{l}\text { Um dia } \\
\text { atrás } \\
n(\%)\end{array}$ & $\begin{array}{l}2-7 \\
\text { dias } \\
n(\%)\end{array}$ & $\begin{array}{c}8-14 \\
\text { dias } \\
n(\%)\end{array}$ & $\begin{array}{c}15 \text { dias } \\
- \\
1 \text { mês } \\
n(\%)\end{array}$ & $\begin{array}{c}\text { Mais de } \\
1 \text { mêss } \\
n(\%)\end{array}$ & $\begin{array}{l}\text { Total } \\
n(\%)\end{array}$ \\
\hline
\end{tabular}

Auxiliar/

técnico de $19(14,3) 11(8,3) 19(14,9) 7(5,7) \quad 17(12,8) 60(45,1) \quad 133(100,0)$ enfermagem

Enfermeiro $\quad 7(8,2) \quad 6(7,0) \quad 13(15,3) \quad 5(5,9) \quad 4(4,7) \quad 50(58,8) \quad 85(100,0)$

Total n(\%) $\quad 26(11,9) \quad 17(7,8) \quad 32(14,7) \quad 12(5,5) \quad 21(9,6) \quad 110(50,4) \quad 218(100,0)$

Considerando o tempo transcorrido desde o último atendimento a pessoa com úlcera vascular, tomando-se como referência os períodos de até sete dias e há mais de sete dias, não houve diferença significativa $(p=0,343)$ entre as categorias profissionais.

Em relação à experiência relatada pelos profissionais de enfermagem na área de avaliação e tratamento de úlceras vasculares (Tabela 3), entre os auxiliares/ técnicos de enfermagem, predominou o relato de "razoável experiência" (51,9\%), enquanto para enfermeiros a maior proporção foi de "pouca experiência" (45,9\%). O autorrelato de "muita experiência" foi referido por menos de $15 \%$ em ambos os grupos.

Tabela 3 - Experiência autorreferida pelos profissionais de enfermagem $(n=218)$ que atuam nas salas de curativos em um município de grande porte da região Centro-Oeste no tratamento as pessoas com úlceras vasculares, set. 2010 a fev. 2011.

\begin{tabular}{|c|c|c|c|c|c|c|c|c|}
\hline \multirow{3}{*}{$\begin{array}{l}\text { Função exercida } \\
\text { na unidade } \\
\text { de saúde }\end{array}$} & \multicolumn{8}{|c|}{ Experiência no tratamento de úlceras vasculares } \\
\hline & \multicolumn{2}{|c|}{ Pouca } & \multicolumn{2}{|c|}{ Razoável } & \multicolumn{2}{|c|}{ Muita } & \multicolumn{2}{|c|}{ Total } \\
\hline & $n$ & $\%$ & $n$ & $\%$ & $n$ & $\%$ & $n$ & $\%$ \\
\hline $\begin{array}{l}\text { Auxiliar/técnico } \\
\text { de enfermagem }\end{array}$ & 49 & 36,8 & 69 & 51,9 & 15 & 11,3 & 133 & 100,0 \\
\hline Enfermeiro & 39 & 45,9 & 34 & 40,0 & 12 & 14,1 & 85 & 100,0 \\
\hline Total & 88 & 40,7 & 103 & 47,3 & 27 & 12,7 & 218 & 100,0 \\
\hline
\end{tabular}

Considerando o nível de experiência em "pouca" e "razoável/muita" experiência segundo a categoria de profissionais de enfermagem não foi encontrada diferença significativa $(p=0,120)$.

No que se refere à realização de curso ou atividade de atualização, segundo o tempo de formação profissional, conforme pode ser visto na Tabela 4, aproximadamente $60,0 \%$ dos profissionais referiram nunca ter realizado um curso ou atividade de atualização relacionada à avaliação e tratamento de feridas. Esse percentual é semelhante levando em conta dos diversos períodos de formação profissional, exceto para aqueles com menor tempo de formação profissional (menos de um ano). 
Tabela 4 - Participação dos profissionais de enfermagem $(n=218)$ em curso e/ou atividade de atualização sobre avaliação e tratamento de feridas, segundo o tempo de formação, set. 2010 a fev. 2011.

\begin{tabular}{lccccccc}
\hline \multirow{2}{*}{$\begin{array}{l}\text { Tempo de } \\
\text { formação } \\
\text { profissional }\end{array}$} & \multicolumn{4}{c}{$\begin{array}{c}\text { Curso/atividade de atualização sobre } \\
\text { avaliação e tratamento de feridas }\end{array}$} \\
& \multicolumn{2}{c}{ não } & \multicolumn{2}{c}{ sim } & \multicolumn{2}{c}{ Total } \\
\hline$<1$ ano & $\boldsymbol{f}$ & $\%$ & $\boldsymbol{f}$ & \% & $\boldsymbol{f}$ & $\%$ \\
$\geq 1<5$ anos & 3 & 33,3 & 6 & 66,7 & 9 & 100,0 \\
$\geq 5<10$ anos & 29 & 61,7 & 18 & 38,3 & 47 & 100,0 \\
$\geq 10<15$ anos & 24 & 60,0 & 16 & 40,0 & 40 & 100,0 \\
$\geq 15<20$ anos & 14 & 57,9 & 16 & 42,1 & 38 & 100,0 \\
$\geq 20$ anos ou mais & 35 & 59,0 & 11 & 40,0 & 25 & 100,0 \\
Total & 127 & 58,3 & 91 & 41,7 & 218 & 100,0 \\
\hline
\end{tabular}

Quanto às instituições que promoveram os cursos e/ou atividade sobre avaliação e tratamento de feridas, conforme referido pelos profissionais de enfermagem, 38 (42\%) participaram de atividades oferecidas pela Secretaria Municipal de Saúde, 19 (21\%) por instituições de ensino superior, 18 (20\%) por hospitais, seis (7\%) por cooperativas médicas, quatro (4\%) em eventos científicos, dois ( $2 \%$ ) por instituições de ensino profissionalizante, um ( $1 \%$ ) por entidades de classe em enfermagem, um (1\%) pela Secretaria Estadual de Saúde, uma (1\%) pessoa não preencheu esta informação e uma (1\%) referiu não se lembrar da instituição que promoveu o curso. Assim, pode-se dizer que a própria instituição empregadora foi a maior responsável pelos esforços de qualificação do pessoal de enfermagem nessa área.

Tabela 5- Temas dos cursos/atividades de atualização em avaliação e tratamento de feridas, realizados pelos profissionais de enfermagem que atendem pessoas com úlceras vasculares, em salas de curativo da rede municipal de uma grande cidade na Região Centro-Oeste, set. 2010 a fev. 2011.

\begin{tabular}{lcc}
\hline $\begin{array}{l}\text { Tema dos cursos/atividades de atualização em } \\
\text { avaliação e tratamento de feridas }\end{array}$ & $\boldsymbol{f}$ & \% \\
\hline Tratamento de feridas & 73 & 80,2 \\
Avaliação de feridas & 24 & 26,4 \\
Classificação de feridas & 15 & 16,5 \\
Técnica de curativo & 10 & 11,0 \\
Anatomia da pele & 07 & 7,1 \\
Cicatrização & 07 & 7,1 \\
Cuidados de Enfermagem & 03 & 3,3 \\
Limpeza da sala de curativos e utilização de & 02 & 2,2 \\
equipamentos de proteção individual & & 1,1 \\
Não lembra & 01 & \\
\hline
\end{tabular}

Em relação à carga horária dos cursos realizados pelos profissionais de enfermagem identificou-se que 53 (59\%) profissionais de enfermagem referiram ter realizado cursos e/ou atividade de qualificação com carga horária menor que oito horas, $16(18,0 \%)$ com carga horária entre 8 a 24 horas, 14 (15\%) participaram de cursos com carga horária maior que 24 horas, quatro $(4 \%)$ referiram não se lembrar da respectiva carga horária do curso e quatro $(4 \%)$ profissionais deixaram o questionário em branco. Dentre os conteúdos abordados nos cursos e/ou atividade de atualização, predominaram aqueles com foco no tratamento da lesão (Tabela 5).

\section{DISCUSSÃO}

Os resultados apontam para um perfil de profissionais semelhante a outros contextos do atendimento de enfermagem, nos quais, se observa o predomínio do gênero feminino e de técnicos e auxiliares de enfermagem em relação aos demais componentes da equipe ${ }^{(10)}$.

Nas unidades pesquisadas predomina no conjunto de técnicos/ auxiliares de enfermagem com idade maior que 41 anos, com tempo de formação profissional maior, em comparação com os enfermeiros, contudo, com tempo de atuação específica na unidade menor que cinco anos, para ambos os grupos. Isso pode ser devido a períodos de contratação cíclicos, nos quais são incorporados tanto novos enfermeiros como técnicos e auxiliares de enfermagem. Admite-se ainda a rotatividade de profissionais, ou seja, os trabalhadores permanecem por um período de tempo na rede municipal, até encontrar oportunidades que eles consideram melhores.

A escala de trabalho de rodízio de locais de trabalho nas unidades básicas, aliado ao pequeno número de enfermeiros que atuavam do modo fixo na sala de curativos, podem trazer implicações negativas no atendimento e acompanhamento das pessoas com úlceras vasculares, e apontam para o não cumprimento de atribuições diferenciadas de acordo com a categoria profissional no atendimento às pessoas com úlceras, contrariando as recomendações nacionais e internacionais ${ }^{(1,11)}$.

O Conselho Regional de Enfermagem do estado de Minas Gerais, Brasil, publicou um documento, constando as atribuições do enfermeiro no atendimento as pessoas com lesões. Elas incluem a responsabilidade por procedimentos técnicos referentes à avaliação e tratamento da pessoa com lesão cutânea, incluindo avaliação da pessoa, indicação do tratamento tópico, realização do procedimento de curativo (limpeza e cobertura) e desbridamento quando necessário. Cabe ao técnico ou auxiliar de enfermagem, segundo esse documento, a realização do curativo (limpeza e cobertura) e desbridamento autolítico e químico, conforme prescrito pelo enfermeiro(11). Assim, compreende-se que a avaliação com o respectivo direcionamento do tratamento tópico seria atribuição do enfermeiro, que desta maneira, teria que prestar cuidados diretos e supervisionar os demais trabalhadores nessa área.

De acordo com a Lei 7.498/86 ${ }^{(12)}$, que regulamenta o exercício profissional de Enfermagem, define, no seu Artigo $4^{\circ}$, que a programação de enfermagem inclui a prescrição da assistência de enfermagem e, assim, todo o tratamento dispensado às pessoas nas salas de curativo deveria ser conduzido mediante a prescrição do enfermeiro. Considerando que a Resolução COFEN 159/1993(13) define em seu Artigo $1^{\circ}$ que 
em todos os níveis de assistência à saúde, seja em instituição pública ou privada, a consulta de enfermagem deve ser obrigatoriamente desenvolvida na assistência de enfermagem, e que a Resolução COFEN 358/2009(14) estabelece a obrigatoriedade da implantação do processo de enfermagem em toda instituição de saúde pública ou privada, depreende-se que as pessoas atendidas nas salas de curativos de unidades de atenção primária à saúde deveriam ter acesso à consulta de enfermagem, derivando dela, o plano terapêutico a ser seguido pela equipe de enfermagem.

Ainda em conformidade com a Lei $7.498 / 86^{(12)}$, no Artigo 14 , fica estabelecido que as atividades do técnico e auxiliar de enfermagem somente podem ser desempenhadas sob orientação e supervisão do Enfermeiro, assim, não seria possível a prestação de cuidado de enfermagem sem o acompanhamento direto desse profissional.

A discreta participação do enfermeiro no tratamento das úlceras vasculares pode contribuir negativamente no processo de cicatrização, manutenção da cronicidade, recidiva das lesões e insatisfação do usuário.

Os dados nessa pesquisa indicam fragilidades no preparo da equipe de enfermagem para o atendimento às pessoas com úlceras vasculares. Foram encontrados dados semelhantes em um estudo com uma população de 20 enfermeiros, entre os quais $18(75 \%)$ consideravam ter pouco conhecimento sobre curativo, embora a metade afirmasse já ter realizado curso ou atividade no tratamento de feridas ${ }^{(15)}$.

Em outro estudo, foi identificado dificuldade das enfermeiras em proceder à avaliação de feridas, a qual esteve relacionada à falta de experiência e de treinamento específico, confirmando a possível insuficiência de conhecimentos a respeito desta temática ${ }^{(16)}$.

Espera-se dos profissionais de enfermagem conhecimentos sobre a fisiopatologia da úlcera de perna, avaliação lesão, uso do Doppler para mensuração do índice tornozelo/braço (IT/B), identificação da cicatrização normal e anormal, seleção do curativo, promoção de cuidados com a pele e com o indivíduo de modo integral, educação para a saúde, prevenção de recorrência e os critérios para encaminhamento à especialista para avaliação ${ }^{(1)}$.

O rodízio dos locais de trabalho, entre os profissionais, pode favorecer a descontinuidade do atendimento às pessoas que apresentam úlceras vasculares, uma vez que, a cada retorno na unidade, a pessoa pode receber orientações e assistência diferentes, uma vez que os profissionais se baseiam em sua experiência clínica para a tomada de decisões terapêuticas.

A escala de local de trabalho fixa e diária poderia contribuir para o alcance de uma assistência de enfermagem com mais visibilidade e resolutividade. Isso permitiria também acompanhar a evolução das etapas do processo cicatricial e a partir destas características da lesão fazer a opção pelo meIhor tratamento e prestar as orientações necessárias, além de promover um maior vínculo entre profissionais de saúde e paciente, importante para promoção da confiança e adesão ao tratamento.

Diretrizes internacionais recomendam que o profissional de saúde tenha treino e experiência adequados em relação aos cuidados às pessoas com úlceras crônicas ${ }^{(1-3)}$. A educação permanente em serviço mostra-se uma ferramenta útil para qualificar o atendimento do profissional de enfermagem contribuindo para uma assistência comprometida, competente e respaldada em conhecimentos teóricos $\operatorname{científicos}^{(17)}$.

A educação permanente permite o desenvolvimento da capacidade de aprendizagem dos profissionais, o desenvolvimento de capacidades docentes e de enfrentamento criativo das situações de saúde, o trabalho em equipes matriciais, a melhoria permanente da qualidade do cuidado à saúde e a constituição de práticas tecnológicas, éticas e humanísticas ${ }^{(18)}$.

Além das atividades de educação permanente, a possibilidade de criação de equipes de apoio matricial e/ou equipe de referência para o atendimento interdisciplinar de pessoas com feridas crônicas poderia favorecer maior eficácia, eficiência do trabalho em saúde. Atendimentos e intervenções conjuntas entre os especialistas matriciais e os profissionais que atuam na assistência às pessoas com úlceras vasculares nas diversas unidades de saúde poderia se configurar em oportunidades de educação permanente.

É possível que o especialista matricial troque conhecimentos e orientações entre a equipe, com diálogo sobre alterações na conduta, o que avaliar no caso e reorientações de condutas antes adotadas, permanecendo o caso sob o cuidado da equipe de referência ${ }^{(19)}$, durante um determinado período, quando então a equipe matricial se deslocaria para outra unidade.

Os cursos oferecidos para atualização dos profissionais parecem estar mais preocupados com a forma adequada do tratamento tópico, não abordando outros temas como a abordagem clínica de enfermagem do cliente, a promoção de um cuidado humanizado, conhecimentos relacionados à avaliação e influência dos fatores psicossociais, psicoespirituais, psicobiológicos no tratamento das pessoas com lesões crônicas.

Além dos conhecimentos sobre os aspectos da fisiopatologia, parâmetros de avaliação da úlcera, critérios na escolha de curativo, orientação do usuário no tratamento e cuidados, os profissionais de enfermagem precisam apropriar-se de conhecimentos relacionados ao cuidado humanizado, a avaliação dos fatores psicossociais e ainda terem a habilidade e sensibilidade de planejar holisticamente o cuidado de modo a contemplar o ser humano em sua plenitude ${ }^{(20)}$.

A assistência às pessoas com úlceras vasculares deve envolver as pessoas que possuem a lesão e seus familiares, numa perspectiva ampliada do cuidado, deve adotar um protocolo de cuidados, tendo como base habilidades e competência técnica e científica da equipe ${ }^{(20)}$.

\section{CONCLUSÃO}

A pouca atuação dos enfermeiros no atendimento as pessoas com úlceras vasculares e a assistência prestada por profissionais com preparo insuficiente pode influenciar negativamente na resolubilidade e no processo de cicatrização destas lesões, contribuindo para manutenção do sofrimento biopsicossocial das pessoas e seus familiares, além de favorecer maior ônus para o sistema de saúde. 
Percebe-se a necessidade de atividades de educação permanente relativas ao atendimento às pessoas com úlceras vasculares e a compreensão por parte dos gestores, sobre os benefícios investimento na capacitação dos profissionais, para o serviço e o melhor aproveitamento dos profissionais já capacitados.
A criação de equipes matriciais para atuarem de modo itinerante nas unidades de saúde poderia contribuir para o aprimoramento dos profissionais durante o seu processo de trabalho, in loco, de modo duradouro e significativo, além de padronizar abordagem e condutas no município no atendimento a essa população.

\section{REFERÊNCIAS}

1. Royal College of Nursing [homepage na internet]. Clinical practice guideline. The nursing management of patients with venous leg ulcers. Recommendations [acesso em 10 mar 2012]. Disponível em: http://www.rcn.org.uk/data/ assets/pdf file/0003/107940/003020.pdf.

2. Conferencia nacional de consenso sobre úlceras de la extremidade inferior (CONUEI). [Evento na internet]. 2009 [acesso em 28 jan 2012]. Disponível em: http://wound. smith-nephew.com/es/node.asp? Nodeld $=3344$.

3. Scottish Intercollegiate Guideline Network [homepage na internet]. Management of Chronic venous leg ulcers: a national clinical guideline. [acesso em 10 mar 2012]. Disponível em: http://www.sign.ac.uk/pdf/sign120.pdf

4. Silva RCL, Figueiredo IBM, Meireles IB. Feridas: fundamentos e atualizações em enfermagem. São Caetano do Sul: Yendis; 2007.

5. Ferreira AM, Bogamif DDD, Tormena PC. O enfermeiro e o tratamento de feridas: em busca da autonomia do cuidado. Arq Ciênc Saúde. 2008;15(3):105-9.

6. Sant'Ana SMSC. Úlceras venosas: caracterização e tratamento em usuários atendidos nas salas de curativos da rede municipal de saúde de Goiânia. Goiânia. Dissertação [Mestrado em Enfermagem] - Universidade Federal de Goiás, Faculdade de Enfermagem/UFG; 2011.

7. Angelico RCP, Oliveira AKA, Silva DDN, Vasconcelos QLDAQ, Costa IKF, Torres GV. Perfil sociodemográfico, saúde e clínico de pessoas com úlceras venosas atendidos em hospitais universitários. Rev Enferm UFPE. 2012;6(1):62-8.

8. Santos MJ, Carneiro LA, Gamba MA. Avaliação da eficácia da pomada de própolis em portadores de feridas crônicas. Acta Paul Enferm. 2007;20(2):199-204.

9. Silva FAA, Freitas CHA, Jorge MSB, Moreira TMM, Alcântara MCM. Enfermagem em estomaterapia: cuidados clínicos ao portador de úlcera venosa. Rev Bras Enferm. 2009;62(6): 889-92.

10. Miyazaki MY, Caliri MHL, Santos CB. Knowledge on pressure ulcer prevention among nursing professionals. Rev Latino-Am Enferm. 2010;18(6):1203-11.

11. Coordenação de Atenção a Saúde do Adulto e do Idoso [homepage na internet]. Protocolo de assistência aos portadores de ferida: revisão [acesso em 28 jan 2012]. Disponível em: http://www.pbh.gov.br/smsa/biblioteca/ protocolos/curativos.pdf
12. Conselho Federal de Enfermagem (Brasil). Lei N 7.498/86, de 25 de junho de 1986. Dispõe sobre a regulamentação do exercício da enfermagem e dá outras providências [legislação na internet]. Diário Oficial da União [acesso em 13 mar 2012]. Disponível em: http://site.portalcofen.gov. br/node/4161

13. Conselho Federal de Enfermagem. Resolução COFEN 159/1993. Dispõe sobre a Consulta de enfermagem [resolução na internet]. Diário Oficial da União [acesso em 13 mar 2012]. Disponível em: http://site.portalcofen.gov. br/node/4241

14. Conselho Federal de Enfermagem. Resolução COFEN-358/2009. Dispõe sobre a Sistematização da Assistência de Enfermagem e a implementação do Processo de Enfermagem em ambiente, públicos ou privados, em que ocorre o cuidado profissional de Enfermagem, e dá outras providências [resolução na internet]. Diário Oficial da União [acesso em 22 jan 2012]. Disponível em: http:// site.portalcofen.gov.br/node/4384

15. Moreira RAN, Queiroz TA, Araújo MFM, Araújo TM, Caetano JA. Condutas de enfermeiros no tratamento de feridas numa unidade de terapia intensiva. Rev RENE. 2009;10(3):83-89.

16. Morais GFC, Oliveira SHS, Soares MJGO. Avaliação de feridas pelos enfermeiros de instituições hospitalares de rede pública. Texto \& Contexto Enferm. 2008; 17(1):98-105.

17. Jacondino $C B$, Severo DF, Rodrigues KR, Lima L, Einhardt RRT, Amestoy SC. Educação em serviço: qualificação da equipe de enfermagem para o tratamento de feridas. Cogitare Enferm. 2010;15(2):314-8.

18. Ministério da Saúde [homepage na internet]. Política e desenvolvimento para o SUS caminhos para a educação permanente em saúde [acesso em 28 jan 2012]. Disponível em: http://bvsms.saude.gov.br/bvs/publicacoes/politica2_vpdf.pdf

19. Dimenstein $M$, Severo AK, Brito M, Pimenta AL, Medei$\operatorname{ros} \mathrm{V}$, Bezerra E. Apoio matricial em unidades de saúde da família: experimentando inovações em saúde mental. Saúde Soc. 2009;18(1):63-74.

20. Torres GV, Mendes FRP, Mendes AFRF, Silva AO, Torres SMSSO, Viana DMO, et al. Cuidados de saúde primários em Évora, Portugal: conhecimento das pessoas com úlceras venosas e avaliação da assistência. Rev Enferm UFPE. 2011; 5(nº esp):360-70. 